Revista Facultad 2019

\title{
Consideraciones acerca del desarrollo de un modelo de educación ambiental emancipatoria para la formación del profesional contable*
}

\author{
Selma Alves Dios ${ }^{\mathrm{a}}$ José Paulo Cosenzab
}

Resumen: Este trabajo presenta una revisión teórica acerca de la integración de la temática ambiental en la educación contable; además, vincula los principios básicos de una sociedad sostenible y discute las limitaciones del modelo convencional de academia y sus reflejos en la formación del contable respecto al conocimiento sobre el medio ambiente. Para esto, se analizan alternativas constructivas de una visión sistémica, crítica e innovadora en el desarrollo académico del profesorado, revertida para la formación del alumnado desde una perspectiva humanitaria y social, que incluye la vida biológica y colectiva de todas las especies como punto central de las preocupaciones con la sociedad y la sustentabilidad. La investigación tiene un carácter cualitativo, en cuanto a la forma de abordaje del problema, y se clasifica como descriptiva en cuanto a su objetivo, y documental por el tipo de procedimiento de investigación. En relación con el tratamiento de los datos, se hizo un análisis de contenido de artículos de investigación publicados en revistas de contabilidad, para producir datos a partir del examen de la naturaleza y del contexto del fenómeno estudiado. El estudio de los resultados muestra que el modelo de educación ambiental dominante no proporciona una formación contable valorando el pensamiento crítico, reflexivo y propositivo, y apunta a mantener las cuestiones económicas, sociales y ambientales en armonía con el desarrollo sostenible y la naturaleza.

Palabras clave: Conocimiento transdisciplinar; desarrollo sostenible; educación ambiental; formación contable; metodología de enseñanza.

Fecha de recibido: 15 de enero de 2019 Fecha de aprobado: 22 de mayo de 2019

Cómo citar: Alves Dios, S. y Cosenza, J. P. (2019). Consideraciones acerca del desarrollo de un modelo de educación ambiental emancipatoria para la formación del profesional contable. Revista Facultad de Ciencias Económicas, 27(2), 127-144. rev.fac.cienc.econ. doi: https://doi.org/10.18359/rfce.3905

JEL: $120, M 41, Q 59$.

* Artículo de investigación. Una primera versión de este trabajo, con el título Proposições para uma formação contábil crítica e ecológica para a construçao de um modelo sustentável de sociedade, se presentó anteriormente en la VIIIth GECAMB 2018 (Portuguese CSEAR - Conference on Environmental Management and Accounting), celebrada en la ciudad de Setúbal, Portugal entre el 8 y el 9 de noviembre de 2018. El presente artículo amplía parcialmente elementos de dicho trabajo, siendo que, en esta nueva versión, intentamos, siempre que sea posible, reconocer las observaciones de los participantes en el referido congreso y también las sugerencias constructivas formuladas por los revisores anónimos.

a Doctora en Contabilidad y Finanzas de la Universidad de Zaragoza, España, magíster en contabilidad de la Fundación Getulio Vargas, Brasil y licenciada en Contabilidad de la Universidad Federal de Río de Janeiro, Brasil. Profesora asociada de la Universidad Federal Fluminense, Brasil. Correo electrónico: selmadios@vm.uff.br ORCID: https://orcid. org/0000-0002-4288-9050

b Doctor en Contabilidad y Finanzas de la Universidad de Zaragoza, España, magíster en Contabilidad de la Universidad del Estado de Río de Janeiro, Brasil, y licenciado en Administración de la Universidad Federal Fluminense, Brasil, y en Contabilidad de la Universidad del Estado de Río de Janeiro, Brasil. Profesor catedrático de la Universidad Federal Fluminense, Brasil. Dirección postal: Rua Mário Santos Braga, s/n, $4^{\circ}$ piso, predio 1 administración, CEP: 24020-140, Niterói, Río de Janeiro, Brasil. Teléfono: +(5521) 2629-9867. Correo electrónico: jpcosenza@id.uff.br oRcid: http://orcid. org/0000-0002-1753-0134 


\title{
Considerations on the Development of a Model for Emancipatory Environmental Education in Professional Accounting Education
}

\begin{abstract}
This paper presents a theoretical review about the integration of environmental issues into accounting education; it also links the basic principles of a sustainable society and discusses the limitations of the conventional academic model and its impact on accountant education related to environmental knowledge. For this purpose, constructive alternatives were analyzed for a systemic, critical and innovative vision in the academic development of faculty, translated into the education of students from a humanitarian and social perspective that includes the biological and collective life of all species as the central point of concerns about society and sustainability. Research is qualitative in its approach the problem, descriptive in terms of its objective, and documentary in the type of research procedure. The contents of research articles published in accounting journals were analyze during data treatment to produce data by examining the nature and context of the studied phenomenon. The study of results shows that the dominant model of environmental education does not provide an accounting education that values critical, reflective and purposeful thinking, and it is intended to maintain economic, social and environmental issues in line with sustainable development and nature.
\end{abstract}

Keywords: Transdisciplinary knowledge; sustainable development; environmental education; accounting education; teaching methodology.

\section{Considerações sobre o desenvolvimento de um modelo de educação ambiental emancipatória na formação do profissional contábil}

Resumo: Este artigo apresenta uma revisão teórica sobre a integração de questões ambientais na educação contábil. Além disso, vincula os princípios básicos de uma sociedade sustentável e discute as limitações do modelo convencional de "academia" e o seu reflexo na formação do contador no que tange o conhecimento sobre o meio ambiente. São analisadas alternativas construtivas de uma visão sistêmica, crítica e inovadora no desenvolvimento acadêmico de professores, revertidas para a formação de alunos de uma perspectiva humanitária e social, que inclui a vida biológica e coletiva de todas as espécies como ponto central das preocupações com a sociedade e a sustentabilidade. Quanto à abordagem do problema, a pesquisa possui caráter qualitativo. Quanto o seu objetivo, é classificada como descritiva e documentada pelo tipo de procedimento de investigação. No que tange o tratamento dos dados, foi realizada uma análise de conteúdo nos artigos de pesquisa publicados em revistas de contabilidade, a fim de produzir dados a partir da análise da natureza e do contexto do fenômeno estudado. O estudo dos resultados mostra que o modelo dominante de educação ambiental não proporciona uma formação contábil que valorize o pensamento crítico, reflexivo e propositivo, e que vise manter as questões econômicas, sociais e ambientais em harmonia com o desenvolvimento sustentável e a natureza.

Palavras-chave: Conhecimento transdisciplinar; desenvolvimento sustentável; educação ambiental; formação contábil; metodologia de ensino. 


\section{Introducción}

Los actuales problemas ambientales, como la agudización del deterioro de los ecosistemas, los cambios climáticos, la sobreexplotación de los recursos naturales, la pérdida de biodiversidad, el calentamiento global, entre otros, son consecuencias indiscutibles de los patrones de comportamiento humano poco amigable con el medioambiente (Moreno Martínez, 2016). Según Pérez Garcés, Victorino Ramírez y Quintero Soto (2016), dichos problemas son resultado de una crisis de civilizaciones, de un modelo económico cuyo estilo de desarrollo y formas de crecimiento económico que se han venido impulsando en los últimos años han depredado a la naturaleza y negado a las culturas alternas.

Sin embargo, de acuerdo con Mora Penagos (2007), los problemas ambientales globales no se tratan solo de implicaciones asociadas al desarrollo económico mundial, sino además, y principalmente, a prácticas de enseñanza y producción de conocimientos académicos que no favorecen modelos de sostenibilidad ambiental. Por lo tanto, es necesario cambiar los patrones actuales de comportamiento humano, que tantos males causan a la vida en el planeta. Esto implica incorporar una nueva actitud general dirigida a consolidar un modelo de civilización sostenible, para preservar el perfecto funcionamiento del medioambiente y motivar el compromiso ético inalienable con su mantenimiento, como valor intangible para las futuras generaciones.

Para la construcción de esta interpretación, hay que generar una nueva ética social que oriente los valores y comportamientos en armonía con los objetivos de la sustentabilidad ecológica y la equidad social desde una perspectiva holística (Organización de las Naciones Unidas para la Educación, la Ciencia y la Cultura [Unesco], 1977). Sin embargo, el modelo de educación ambiental predominante no favorece la efectividad de esta visión, en la medida en que está sujeto, en su forma y contenido, a las limitaciones del pensamiento en la misma academia, ya sea como transmisora de conocimiento o, principalmente, como generadora de este.
El problema de ese estudio surge de la pregunta: ¿qué condiciones en el pensamiento y en las prácticas académicas pueden estar impidiendo el desarrollo de una formación efectivamente direccionada para la sustentabilidad del planeta y de la humanidad? En este marco, el presente artículo tiene dos objetivos principales: 1) identificar las limitaciones del modelo convencional de la academia que conduce a un comportamiento pragmático y utilitarista en la visión de mundo y 2) discutir sus reflejos en la manera como el conocimiento ambiental es desarrollado y transmitido en la enseñanza universitaria en contabilidad.

Para examinar el problema, se buscan visiones desde afuera de la contabilidad, de diversas áreas de conocimiento e iniciativas y proposiciones de otros tipos de conocimiento; además, quiere plantear visiones alternas para transmutación del alma académica ante las limitaciones de la racionalidad económica e instrumental. Para este fin, la aplicación metodológica realizada empleó un abordaje cualitativo, llevando a cabo un acercamiento al pensamiento científico sobre el tema soportado en una revisión documental en la base de datos Scopus; esto, con el objetivo de formular una reflexión epistemológica crítica sobre la manera tradicional de enseñanza del conocimiento ambiental y plantear un modelo de educación ambiental en línea con el desarrollo de la contabilidad como ciencia social. El uso del análisis de contenido (Bardin, 2016) permitió mirar analíticamente dicha producción científica, para darle la creación de categorías con base en los datos (Pérez, 1994) y posibilitar la interpretación del sentido oculto de los hechos bajo un proceso de interacción entre los textos y contextos donde están planteados, señalando algún conjunto de reglas para el análisis (Abela, 2000).

En síntesis, se espera contribuir a la construcción de una visión sistémica, crítica y alterna en el desarrollo académico del profesorado, que sea revertida en la formación del alumnado de contabilidad desde una perspectiva humanitaria y social que incluya la vida biológica y colectiva de todas las especies, como punto central de las preocupaciones que se relacionan con la sociedad y la sostenibilidad. 
El estudio está dividido en cinco secciones, incluida esta introducción. Después de esta se presenta una visión de la conformación del pensamiento y las prácticas en el modelo actual de academia, señalando los paradigmas subyacentes y sus implicaciones en dicho modelo y en el campo ambiental. Después se menciona el papel y los requisitos de la educación ambiental, abordando las exigencias de visión crítica y bien como de proposiciones en educación ambiental. Luego se comentan los desafíos y las proposiciones para un modelo de educación ambiental en contabilidad, identificando nexos entre la contabilidad y el contexto medioambiental, y presentando propuestas para una educación ambiental renovada en la formación contable. Finalmente, están las conclusiones y consideraciones finales sobre el tema, a las que les siguen referencias bibliográficas utilizadas en el estudio.

\section{Conformación del pensamiento en el modelo actual de academia y las implicaciones en el contexto ambiental}

Los estudios críticos más recientes, en el campo de la educación, han reiterado que el pensamiento científico viene siendo capturado como instrumento que está al servicio de un modelo de academia que privilegia la dominación y rechaza la formación emancipadora, orientada a contribuir con la transformación de una realidad que, históricamente, se pone en contra a la construcción de sociedades justas y armónicamente equilibradas social y económicamente.

Partiendo de la idea de que la educación se presenta como el componente clave para el logro de un desarrollo sostenible (Unesco, 2014), se hace imperante discutir los paradigmas científicos dominantes y sus implicaciones en la academia y en la vida de las personas, las economías de los países y nuestro mundo en general.

\section{Paradigmas subyacentes a la ciencia moderna}

El modelo actual de academia, tanto en su forma como en su contenido, está condicionado por una manera de ver el mundo, que se estableció casi inconscientemente y que se aplica en todas las ciencias. Según Arntz, Chasse y Vicente (2007), en la antigüedad se creía en un mundo vivo, donde los pueblos de cada continente vivían una relación armoniosa con su entorno - los animales, las plantas, el Sol y la lluvia, la tierra viva-. La manifestación de esa percepción ocurría por medio del descubrimiento de "espíritus" en montañas, arroyos y bosques en todas estas culturas. El objetivo de la ciencia era, entonces, adquirir conocimientos que permitiesen armonizar la vida humana con las grandes fuerzas del mundo natural y los poderes trascendentales que estos pueblos presentían que existían más allá del mundo físico. Capra y Luisi (2016) señalan que desde los tiempos de los antiguos, los objetivos de la filosofía natural habían sido la sabiduría, la comprensión del orden natural y la vida vivida en armonía con ella.

Esta dinámica de pensamiento fue reprimida en el periodo del oscurantismo, cuando la Iglesia tenía el control de la generación y divulgación del conocimiento. Entre los científicos, los esfuerzos de investigación se restringieron a la materia y culminaron en las formulaciones que condujeron al surgimiento de la ciencia moderna, donde la noción de un mundo espiritual, orgánico y vivo fue sustituida por la concepción del mundo como una máquina, como propuso Descartes. $\mathrm{Al}$ anunciar los descubrimientos y las teorías científicas desde un contexto de fuerte oposición a la religión, los hombres de la ciencia utilizaban gran parte de su energía mental intentando probar la falsedad de las afirmaciones religiosas. Ante ese clima mental, el péndulo de la investigación de la "verdad" osciló exclusivamente la invariable creencia en la "razón" y en la "lógica", aprisionando el intelecto humano al materialismo en la búsqueda de respuestas sobre los orígenes de la vida y de la existencia (Cartas de Cristo, 2012).

Los esfuerzos de los científicos pasaron entonces a describir cuantitativamente la naturaleza, lo cual se extendió del estudio de la materia al de todos los fenómenos naturales y sociales; esto consolidó la visión mecanicista del mundo, compuesto de partes en funcionamiento y explicable por leyes previsibles y mecánicas (Capra y Luisi, 2016). De 
esa forma, solo puede ser objeto de la ciencia y, por tanto, del cuerpo de conocimiento real aquello que se puede medir. Con Francis Bacon la ciencia comenzó a representar un medio en busca del dominio del hombre sobre la naturaleza y se estableció el carácter de objetividad de la ciencia experimental.

Esa visión fue compartida por educadores, filósofos y estadistas de su tiempo, y la educación escolar comenzó a buscar la difusión de este nuevo conocimiento. En este sentido, la ciencia pasó a ser concebida por la comunidad científica como la única forma de conocimiento auténtico, y de esta manera conquistó la hegemonía sobre los demás tipos de saberes.

\section{Implicaciones en el actual modelo académico}

La asunción de que el conocimiento científico es la única forma de conocimiento verdadero induce a la idea de que ese tipo de conocimiento pueda ser la representación más cercana a la realidad. Sin embargo, muchas "verdades" científicas ya fueron descartadas a lo largo de la historia, aunque han podido causar muchos daños al ser humano o a la naturaleza. Por ejemplo, la lobotomía, una práctica hoy prohibida, ha sido considerada un gran avance en la cura de los problemas mentales. Por otro lado, el método científico no es capaz de conocer todo lo que existe, ni de entender sus relaciones, ni de lidiar con realidades que no son cuantificables o mensurables. Además, el planeta Tierra es apenas un caso entre una infinidad de cuerpos celestes semejantes; es decir, es una muestra poco representativa para que se pueda comportar con seguridad ante las conclusiones científicas obtenidas hasta el momento, en particular respecto a las reacciones de la naturaleza a las intervenciones de los humanos.

La hegemonía del conocimiento científico también lleva al descarte de toda forma de conocimiento ajena al método científico, es decir, todos los demás tipos de saberes, y de esta forma priva la ampliación del universo de fenómenos y objetos pasibles de comprensión. Según Pérez Garcés et al. (2016), con la ayuda de grandes avances tecnológicos la civilización moderna fue distanciando de manera gradual a los seres humanos del dominio real de sus medios de subsistencia más básicos, y los hizo creer, paradójicamente, que cada vez dependían menos del ambiente natural. En el caso de la medicina, por ejemplo, el pensamiento convencional se basa únicamente en la intervención de fármacos, despreciando el poder de formas mucho más antiguas de cura, llamadas - erróneamente en nuestra visión- "alternativas". Sin embargo, los otros tipos de saberes son importantes justamente por no estar limitados a los estrictos paradigmas de la ciencia moderna. En América Latina es todavía más acentuado, ya que los conocimientos ancestrales fueron reemplazados por una cultura eurocéntrica, impregnada de la lógica de exploración de los seres humanos y de la naturaleza y desprecio por los saberes locales.

El énfasis en la cuantificación hizo que aquello que no se puede medir haya quedado sin atención por parte de la ciencia, lo que privó al ser humano de la comprensión de los aspectos inconmensurables de la existencia y sus propiedades esenciales, como las emociones, las intenciones, los sentimientos y el sentido de la vida. Según Santos (2010), en la ciencia moderna el conocimiento basado en la experiencia no tiene el mismo reconocimiento como evidencia empírica, que recibe una lógica de investigación de las matemáticas. Esto genera dos consecuencias: 1) el rigor científico sobre las mediciones, es decir, "conocer significa cuantificar", y 2) la reducción de la complejidad, es decir, en vista de la complejidad de los hechos del mundo, la mente humana no es capaz de comprender el fenómeno de una manera total, por lo que "saber significa dividir y clasificar".

Por esta razón, la visión mecanicista lleva a la forma compartimentada de generación y difusión del conocimiento, en la cual se restringe la comprensión de los fenómenos de la existencia al prisma de un determinado campo de conocimiento, y se priva de esta manera la visión desde otras perspectivas. Sin embargo, aunque todos los fenómenos - en particular los sociales - se derivan de diversos fenómenos e interacciones, identificables en diferentes áreas de conocimiento, cada uno tiende a ser analizado desde una perspectiva limitada a su estructura conceptiva dirigida a sus 
percepciones. Esto compromete inexorablemente la capacidad de percepción ecológica de la interdependencia fundamental de todos los fenómenos; además, no permite comprender los problemas en sus diversas dimensiones ni presentar propuestas de soluciones sistémicas para atender a las necesidades de todos y así perfeccionar el bienestar colectivo. En la medicina, cada órgano del cuerpo tiene su funcionamiento y es visto de forma especializada y aislada como enfermedad; de igual forma, no se considera la conexión cuerpo-mente ni los efectos de las emociones y pensamientos positivos en nuestro cuerpo.

La idea de que solo es real aquello que puede ser visto o medido, además de reforzar el "enyesado" positivista y demandar las comprobaciones que priorizan algún modelo cuantitativo, es negar la posibilidad de cualquier fenómeno más allá del mundo visible. Esto restringe las proposiciones sobre lo nuevo y las nuevas formas de organizarse colectivamente, de tratar a los seres vivos; asimismo, ignora la inteligencia detrás de los ecosistemas, incluso en las ciencias sociales. Como mencionan Arntz et al. (2006), "si todo lo que percibo se basa en lo que ya sé de antemano, ¿cómo voy a percibir algo nuevo? Y si nunca percibo nada nuevo, ¿cómo voy a cambiar?, ¿cómo voy a crecer?" (p. 23).

Así, la lógica de la dominación sobre la naturaleza termina por justificar a tantas gestiones y creaciones, en cualquier área de conocimiento que sea, que han llevado al planeta a las condiciones de degradación en las que se encuentra; de esta forma, ha influido, incluso, en la dinámica de tratamiento de la cuestión de la sostenibilidad.

\section{Negación de la dimensión política}

En el marco de las circunstancias determinantes del problema de la sostenibilidad en el planeta, quizás la más relevante sea la dimensión política y la ideología subyacente. Es por la acción política y, más específicamente, por su "sesgo ideológico" que se determina la insostenible forma de civilización que se tiene hoy. Incluso, las mismas limitaciones intelectuales y técnicas de la forma de pensar, sedimentadas después de la revolución científica, se agravan por el hecho de que el "diseño científico" del pensamiento en la academia no prevé la existencia de la dimensión política y de una ideología subyacente a los pensamientos y las prácticas en la vida cotidiana, en la economía y en la misma academia.

La negación de la dimensión política ocurre principalmente de forma velada, ya sea por la narrativa de los fenómenos de manera aislada, sin considerar el contexto de su incidencia y las motivaciones inherentes, o por la falta de estímulos a la expresión crítica, cuya evidencia es la casi nula incidencia de investigaciones y proposiciones que se encuadran en esos perfiles. Asimismo, no se observa una actuación expresiva de la academia para cohibir acciones negativas y omisiones de aquellos que tienen la responsabilidad o detienen los medios para ayudar a cambiar la situación de extrema pobreza con la que vive la mayor parte de la población mundial, y estancar la violenta degradación ambiental del planeta.

La falta de visibilidad de la dimensión política puede deberse también a que no sea muy evidente en los fenómenos, sino que su práctica es a menudo oculta. Según Stiglitz (2006), las organizaciones dedican esfuerzos sustanciales, frecuentemente ocultos, para definir las reglas de los mercados donde actúan. Es probable, en ciertos casos, que esté relacionada con iniciativas que van en contra de los intereses colectivos y que promueven la degradación del medio ambiente.

En el contexto de la educación, además de la negación de la dimensión política, la influencia política puede ocurrir incluso directamente en el propio proceso de definición del modelo de educación formal que se ofrece hoy, desde la enseñanza fundamental hasta el posdoctorado, ya sea por la forma como se determinan los gestores, por los cambios en los currículos y contenidos pedagógicos o por la definición de los asuntos que serán investigados. Por ejemplo, el Banco Mundial y el Fondo Monetario Internacional cuando intervienen en la organización política y económica de los países también suelen intervenir en las propuestas educativas, a través de acuerdos de "cooperación" internacional.

En el contexto de sostenibilidad y de justicia social, la dimensión política se disfraza en la 
retórica de las declaraciones de compromiso con la búsqueda del desarrollo sostenible, mientras permanecen imputando pérdidas a la sociedad y profundizando la desigualdad social, en especial por parte de las grandes corporaciones (Cosenza, Ribeiro y Dios, 2017). El Pacto Global de las Naciones Unidas es un ejemplo de retórica legitimada por iniciativas de mucho impacto en los medios de comunicación colectiva, pero de poco efecto práctico. Aunque su alcance aborde grandes frentes como derechos humanos, derechos laborales, medio ambiente y lucha contra la corrupción (Gómez Patiño, 2011), se observan, en la práctica, iniciativas de agentes privados que "capturan" el Estado y las empresas, en vista del nivel de corrupción que debilita la estructura económica de algunos países, especialmente los menos desarrollados. Tal actitud influye en la realidad social de dichos países, además de distorsionar en la formulación de leyes, normativos, reglamentos y políticas públicas, de tal forma que se favorecen los intereses de esos grupos dominantes, que egoístamente se van en detrimento del bienestar general de las sociedades (Garay Salamanca, Salcedo Albarán, León-Beltrán y Guerrero, 2008).

\section{La ideología del fundamentalismo de mercado}

Aunque el funcionamiento de la economía, en su forma actual, genere los problemas sociales y ambientales en la magnitud que se encuentran hoy, como la generalización de la injusticia, la contaminación del aire, la escasez de agua dulce, las actividades perniciosas, el valor de la vida humana e incluso los conflictos armados, no se contemplan en las políticas oficiales medidas efectivas sobre los límites para la maximización individualizada de la riqueza, ni sobre las pérdidas colectivas de esa forma de actuar.

En tal posicionamiento se alinean los intereses predominantes del libre funcionamiento del "mercado", sin control de las relaciones entre los agentes de la economía. Subyacente a esa visión se identifica el paradigma del fundamentalismo de mercado en el cual, según Stiglitz (2006), individuos coherentes, con expectativas racionales actúan en mercados perfectamente competitivos junto con empresas también competitivas que obtienen beneficios y que han proporcionado el marco dominante de la conducta de las ciencias sociales y del estudio de las organizaciones, ya que la mayoría de los teóricos de la organización, sociólogos y psicólogos industriales abordan sus temas dentro de esta perspectiva. Para Tirole (2018), ese paradigma se ha vuelto el modelo dominante, si no único, de la organización de las sociedades contemporáneas.

La condición ideológica en la asunción de ese paradigma, es decir, que el "mercado" se comporta de forma racional, se evidencia por el hecho de que aunque se tratara de una formulación emanada en el campo teórico de la economía (y, por tanto, no cumple los mismos prerrequisitos de conocimiento científico), logra tener un estatus de verdad en medida significativa de la academia, adoptado como conocimiento legítimo en la enseñanza para respaldar análisis o de forma indirecta por la aceptación acrítica de sus lógicas y prácticas derivadas. Una explicación para la aceptación inerte de esa lógica se encuentra en Silva (2019), para quien la "escasez" que se presenta en la teoría económica como un "paradigma natural" descalifica el análisis crítico de sus nefastos desdoblamientos teóricos y concretos, y favorece así su perpetuidad. Con base en esto, por ejemplo, se ha asentado la idea de que alcanzar cada vez más ganancias y proporcionar mayor rentabilidad a las empresas y sus accionistas es un comportamiento racional.

\section{Implicaciones en el ámbito medioambiental}

Capra y Luisi (2014) afirman que una comunidad humana sostenible debe ser planeada de tal forma que sus vidas, negocios, economías, estructuras físicas y tecnologías no interfieran en la capacidad inherente de la naturaleza para sostener la vida. El bienestar y la supervivencia de la humanidad dependen fundamentalmente de la capacidad de vivir de acuerdo con la comprensión de los principios básicos de la ecología -la organización de los ecosistemas para sostener la red de la vidaasí como de una profunda percepción ecológica de la interdependencia fundamental de todos los 
fenómenos. Para ello, es necesaria la concientización de que el ser humano está inserto en los procesos cíclicos de la naturaleza. Todo ello con el fin de desarrollar los parámetros de vida, a lo largo del tiempo, en continua interacción con los otros sistemas vivos, humanos y no humanos.

Sin embargo, en términos generales, la gestión ambiental se orienta esencialmente hacia el intento de delimitar la relación "consumo" de recursos naturales y "calidad" de vida de la sociedad; es decir, se basa en el establecimiento de un equilibrio entre lo que la naturaleza puede ofrecer y lo que efectivamente podría ser utilizado por la población en la mejora de sus condiciones de vida. No obstante, de acuerdo con Leis (1991), esa perspectiva ambientalista tiene un núcleo contradictorio y engañoso, porque permite creer que la humanidad puede establecer una relación de equilibrio con la naturaleza sin devolverle o reconocerle un papel activo y creativo; de esta manera busca adaptar el proyecto ecológico al proyecto de sociedad dominante en nuestra civilización contemporánea.

Esto se refleja en el carácter institucional oficial, o sea, entre los organismos supranacionales con reconocida preponderancia en al tratamiento de ese problema, dada la inexistencia de un documento lato o la falta de consenso sobre la mejor forma para hacer sostenible una sociedad. Como describe Veiga (2012), el dilema de intentar reconciliar la perennidad del crecimiento de la actividad económica con el concepto de desarrollo sostenible no es ni siquiera reconocido en las contribuciones dadas a la United Nations Conference on Sustainable Development (UNCSD-2012) y a la Río+20 (Conferencia de Desarrollo Sostenible de Naciones Unidas). Eso ocurre, incluso, en el marco de documentos desarrollados por organizaciones internacionales, aunque usen las expresiones economía verde (United Nations Environment Programe [PNUMA], 2011) y crecimiento verde (Organización para la Cooperación y el Desarrollo Económicos [OECD], 2011). Esto es más evidente todavía en el Informe Spence, The Growth Report - Strategies for Sustained Growth and Inclusive Development (Banco Mundial, 2008). Así, las proposiciones en el campo de la sostenibilidad tienden a limitarse a soluciones ecológicas concretas, sin profundas alteraciones sistémicas en la forma de vida en sociedad.

Por otro lado, no hay aún ninguna medida objetiva, a nivel supranacional, para que los países pasen a adoptar los principios de ecología; por ejemplo, el uso de la energía solar, que por la amplia gama de beneficios sociales y ambientales debería ser tratado como la primera medida concreta en la dirección de la sostenibilidad. En contraste, las iniciativas en torno a la explotación de combustibles fósiles son abundantes, sean técnicas, legales u operativas, en el sector público, privado o en el propio ambiente académico.

La misma política ambiental mundialmente acepta se presenta dentro del paradigma del fundamentalismo de mercado, al sustentar un modelo de desarrollo sostenible que parte de la idea errónea de buscar armonizar el proceso económico con la naturaleza privilegiando "un modo de producción y un estilo de vida insustentables que se han vuelto hegemónicos en el proceso de globalización" (Pérez Garcés et al., 2016, p. 5). El reduccionismo que gobierna el mundo moderno se constituye en una sumisión de los factores políticos y sociales a los económicos y de esos a los financieros, lo que promueve, según Chanlat (2002), una lógica de vida con la cual el dinero pasa a jugar el papel principal en el desarrollo y en la sostenibilidad del planeta.

Esa forma de pensar es permisiva con el modelo de economía desarrollista, que conduce a un crecimiento económico exacerbado y predatorio, tanto de los recursos ambientales, como de los diversos tipos de seres, incluido los humanos. Así, a pesar de estimular de algún modo los saberes y el hacer en educación ambiental y sustentabilidad, los conocimientos y las convicciones repasadas en todas las áreas de conocimiento acaban inhibiendo el cambio radical de la forma como el modelo contemporáneo de civilización se desarrolla.

\section{Papel y requisitos de la educación ambiental}

Para Vásquez Vargas (2014), “una educación que promueve el desarrollo sostenible significa una educación que aborde planteamientos metodológicos que permitan un cambio de mentalidad y 
de actitud de las personas" (p. 8). Por lo tanto, la educación ambiental para el desarrollo sostenible exige métodos de enseñanza y aprendizaje que motiven un pensamiento crítico y transformador en la formación de los alumnos. Para esto, debatir y reflexionar respecto al papel y los desafíos concernientes a la educación ambiental alineada a los objetivos de desarrollo sostenible es importante como herramienta de transformación epistémica y socioecológica para alcanzar un desarrollo sostenible (Collado-Ruano, 2017, p. 244).

\section{Papel de la educación ambiental}

Los profundos cambios en la organización y en el modo de vida de la sociedad moderna dependen de una consciencia general de la necesidad y puesta en marcha de una educación transformadora, que lleve a una verdadera revolución en los ideales de existencia y a una nueva forma de relacionarse con el cosmos y todos sus elementos.

El papel de la escuela en la educación para el desarrollo sostenible, según Rodríguez Villamil, Guerra García y Guzmán Cómbita (2011), se fundamenta en el hecho de que la escuela, además de ser una entidad donde se refleja y se crea una cultura, tiene la capacidad de transformar profundamente las estructuras sociales; es decir, la forma de pensar, sentir y actuar de los individuos. Con ese propósito, la educación para el desarrollo sostenible promueve una ciudadanía responsable y, por lo tanto, "la educación ambiental va mucho más allá del logos, pues también concibe el conocimiento como el instrumento que le permite al hombre "racionalizar" sus actitudes hacia el espacio que lo rodea y los recursos que hacen parte de la naturaleza" (Rodríguez Villamil y Guerra Guerra, 2009, p. 77).

Para Dias (2013), a la educación ambiental le compete ejercer un papel politizado, entrelazado a los problemas ambientales y sociales, para contextualizar, discutir e integrar las acciones ambientales a los intereses de las poblaciones y de las clases sociales más afectadas por los problemas socioambientales. Una educación ambiental que promueve el desarrollo sostenible significa, según Roças y Anjos (2014), impartir un modelo educacional que busca el despertar de la ciudadanía, estimulando el desarrollo de una forma de pensar basada en el raciocinio lógico y crítico, y en una memoria histórica; es decir, construido en un contradiscurso a la base hegemónica, al propalar un nuevo modo de vida a la sociedad, dentro de un nuevo modelo político y social.

Según Pérez Garcés et al. (2016), el gran reto que se plantea es:

[...] generar un proceso orientado a la sustentabilidad fundado en el reconocimiento de los límites y potenciales de la naturaleza, así como la complejidad ambiental, para promover una nueva alianza naturaleza-cultura capaz de crear una nueva economía, reorientar los potenciales de la ciencia y la tecnología, y construir una nueva cultura política fundada en la ética de la sustentabilidad (p. 5).

Para Rojas López, Uribe Giraldo y Roja Valencia (2018):

[...] la educación es el motor del cambio organizacional y social, por lo que el desarrollo sostenible requiere de una estrategia educativa que transforme los actuales estilos de vida, la civilización consumista y las costumbres que hacen que la realidad actual sea insostenible (p. 257).

En otras palabras, la educación es vital para el cambio individual y social (Unesco, 1980; Unesco y Programa de las Naciones Unidas para el Ambiente [UNEP], 1985).

\section{Visión crítica y actitud como requisitos fundamentales}

Las proposiciones de educadores y activistas comprometidos con la búsqueda de soluciones para el problema de la sostenibilidad señalan, en su grande mayoría, la necesidad de profundos cambios en el comportamiento y en el modo de vida de la sociedad moderna. Sin el cambio profundo de la mentalidad instrumental-tecnológica-consumista predominante, el escenario futuro más probable será el colapso ecológico total (Leis, 1991). Por ejemplo, la puesta en marcha de la moderna conciencia ambiental se ha dado a partir de la actuación crítica de la bióloga marina y conservacionista estadounidense Rachel Louise Carson (1907-1964), en la cual se advierte sobre los efectos nocivos al 
medio ambiente y a los seres humanos causados por el uso indiscriminado de pesticidas sintéticos (sobre todo el dicloro-difenilo-tricloro-etano, conocido por la sigla DDT). En 1962, la publicación del libro Primavera silenciosa, de Carson, llevó a un nivel sin precedentes la preocupación sobre el medio ambiente en la conciencia colectiva de la sociedad estadounidense; asimismo, la educación ambiental tuvo impulso y tras una década el uso del DDT fue prohibido en Estados Unidos.

Así, es imprescindible la adopción de una formación crítica, capaz de promover la percepción de las paradojas actuales en cuanto a la manera de conducir el problema de la insostenibilidad de la vida en el planeta, por parte de los organismos oficiales, economistas clásicos y líderes empresariales globales, así como por los políticos e incluso por los sistemas formales de educación superior. Hay que considerar y comprender que la dimensión política impregna a todos los fenómenos y a los diversos mecanismos que disimulan los intereses de mantener el modelo actual de explotación económica y de concentración de poder y riqueza, subyugando a ellos la calidad de vida y bienestar de las poblaciones y a la preservación de la naturaleza. Tras este reconocimiento, no parece exagerado afirmar que el enfoque hoy dominante de la educación para el desarrollo sostenible, incluso acaba determinando lo que la academia debe saber y su comportamiento.

Según Leff (1996, 1995 y 1994), la racionalidad ambiental implica una nueva ética y nuevos principios de producción que van más allá de la racionalidad económica actual. Eso supone repensar, por ejemplo, la complejidad del pensamiento que problematiza las ciencias en la incorporación del saber ambiental emergente. La racionalidad ambiental requiere un programa de educación ambiental democráticamente comprensivo y complejo, abierto a un amplio espectro de actividades $\mathrm{y}$ actores.

Para Capra y Luisi (2014), el aprendizaje debe convertirse en una habilidad crítica para todos los ciudadanos, pero también tiene que alcanzar principalmente a los líderes empresariales, los profesionales de todas las esferas y los políticos, para convertirse así en la parte más importante de la formación ambiental, en especial en el nivel universitario, donde ciertos tipos de conocimiento y valores se enseñan a los líderes del mañana. Sin embargo, Quinche Martín (2017) señala que

[...] la sostenibilidad fuerte no será posible si no hay una transformación de las relaciones sociales predominantes centradas en lo económico financiero más allá de la participación política y el reconocimiento de las particularidades y las diferencias entre las sociedades y dentro de ellas (p. 163).

Urge entonces la construcción de un discurso del conocimiento y de la educación que, además de tener sinergia con la forma de entender y abordar los problemas ambientales, vaya más allá del aprendizaje acerca del medioambiente y busque así una articulación del pensamiento ambiental configurada en una visión sistémica, compleja e interdisciplinaria (Mora Penagos, 2012). Para dar solución a esto se necesita que el paradigma de la modernidad tecno-científica sea analizado, cuestionado y reconstruido. Así, la educación ecológica debe ser concebida como una herramienta de racionalidad ambiental, en sustitución del dominante modelo de racionalidad económica.

\section{Proposiciones de educación ambiental}

Un modelo de educación ambiental no puede quedarse restringido solo a los "efectos" de los problemas causados por la degradación ambiental (Dias, 2013), pero sí exponer sus “causas", y de esta forma evidenciar las relaciones de poder y dominación que engendran las sociedades contemporáneas (Guimarães, 2000).

El saber ambiental debe connotar los saberes marginados y subyugados por la centralidad del logos científico, en contrapunto con la racionalidad económica, científica y tecnológica de la modernidad. En ese sentido, la multidisciplinariedad es una condición inherente a la ciencia de la ecología. Una vez que los ecosistemas interconectan el mundo vivo con el mundo no vivo, la ecología necesita fundamentarse no solo en la biología, sino también en la geología, la química atmosférica, la termodinámica y otras ramas de la ciencia (Capra y Luisi, 
2014). Por otro lado, cuando se trata de evaluar los impactos de las actividades humanas sobre la biósfera - lo que cada vez más urgente-, se debe mencionar a la ecología, una gama totalmente nueva de campos que incluyen la agricultura, la economía, la planificación industrial y la política.

Luego, está el denominado estímulo a la visión de mundo como ente vivo, es decir, ver al planeta como un organismo que siente y reacciona a las acciones humanas. Esta forma interconectada de observar al mundo, por ejemplo, permitió que Emoto (1999) identificara las reacciones del agua a los gestos humanos en los cristales de agua. Mirar nuestro mundo desde esa perspectiva exige la utilización de un referencial teórico surgido en los postulados de la teoría histórico-crítica (Bonfim, 2008, 2011; Deluiz y Novicki, 2004; Dias y Bonfim, 2011; Loureiro, 2004, 2007, entre otros), de manera que se pueda formular una posición contraria que se presente como una alternativa diferente al modo de desarrollo socioeconómico que fue construido en los últimos siglos (Dias, 2013).

Leff (2002) señala que es necesario romper las fronteras de los dominios conceptuales y materiales de las disciplinas, derrotando las estrategias del poder implícito en el pensamiento y en la complejidad de los métodos, abriéndose a otras racionalidades, no disciplinarias, en la dirección del diseño y de la conducción de un programa interdisciplinario, cuya enseñanza sea algo más que la simple conjugación de asignaturas en el plan de estudios. Para eso, es importante desarrollar un programa multitemático en el campo socioambiental, donde se construirán nuevos saberes, técnicas y conocimientos (inscritos en un contexto de creación científica), que serían incorporados como contenidos integrados en el proceso de formación del alumnado. Eso requiere la construcción de nuevos objetos interdisciplinares de estudio, a través de la problematización de los paradigmas imperantes, de la formación de los docentes y de la incorporación del saber ambiental emergente en nuevos programas curriculares de la enseñanza universitaria en contabilidad (Leff, 2008).

En la ausencia de iniciativas institucionales que promuevan los cambios necesarios en la conformación de las futuras generaciones y en el repase de los saberes, cada persona tendrá que tomar las riendas de su aprendizaje en la comprensión de los principios básicos de ecología aplicados a las comunidades humanas. Un camino para esto es identificar experiencias relevantes que se identifiquen con principios básicos de ecología, haciendo constar los saberes en la materia que será transmitida. Se puede también pensar en la formación de alianzas entre docentes de áreas diferentes de conocimiento, para desarrollar formas de rescate y soporte al desarrollo y aplicación de saberes relevantes para una vida efectivamente sostenible, feliz y sana.

\section{Desafíos y proposiciones para un modelo de educación ambiental en contabilidad}

La educación ambiental se pone en la actualidad como un derecho fundamental y la base del progreso de cualquier país (Unesco, 2014). Sin embargo, para ser efectivo, dicho modelo educacional necesita ser planteado desde el enfoque holístico, de modo que garantice una educación inclusiva, equitativa y de calidad, y promueva oportunidades de aprendizaje durante toda la vida para todos (Unesco, 2017).

Como parte de este reto, la contabilidad puede contribuir al desarrollo sostenible incorporando un modelo de formación del profesional contable que ampara la investidura del conocimiento científico, y fortalece así los principios y valores que orientan la preservación del medioambiente. Para eso, hay que revisar los contenidos enseñados en la disciplina de contabilidad ambiental, de manera que se puedan construir propuestas adheridas con los conceptos de responsabilidad social empresarial y desarrollo sostenible, conjuntamente.

\section{La contabilidad y el contexto medioambiental}

El papel que juega la contabilidad en la dimensión política se plantea desde su capacidad de contextualizar las relaciones de poder, no solo como fuente eminente de información, en ese caso poseedora de poder, sino también como objeto de poder, al convertirse en rehén de directrices y 
políticas impuestas por entidades reguladoras de la actividad contable (Cosenza, Cardoso Teixeira y De Souza Sant'Anna, 2012). Al nutrirse de un conjunto de preceptos y principios que conducen los comportamientos y las acciones individuales y colectivos, la contabilidad puede despertar el interés de los agentes económicos por aspectos inherentes a las prácticas contables, de modo que influya en las relaciones de poder en las organizaciones y en la sociedad en cuanto a la dimensión ambiental.

A lo largo del tiempo, la contabilidad siempre ha ayudado a sustentar el statu quo dominante (León Martínez, 2008), y se ha orientado a la lógica de la racionalidad económica/tecnológica del capitalismo que moldea la explotación de las personas y del medioambiente. El sistema capitalista y las estructuras organizativas de las empresas que de él emergen se convierten en formas de racionalización que conducen la contabilidad a revelar una calificación de la realidad observada bajo el cribado de la "objetividad". Con ello, el contador dibuja y proyecta, como problema gnoseológico, una visión objetivista y técnica del conocimiento, considerando pertinente todo lo que se sea útil para la eficiencia, el control y la gestión del capital, en una especie de "régimen de verdad" de manera foucaultiana (León, 2008).

Tanto técnica como científicamente, la discusión teórica de la contabilidad, como campo de conocimiento social aplicado, se configura a partir de una vinculación que mide el beneficio con base en principios de racionalidad socioeconómica que está articulado por la base de ganancias de ese modelo dominante.

Según García Casella y Rodríguez Ramírez (2001):

[...] integran el dominio o universo del discurso contable todos los componentes, ya sean objetos, hechos, personas o reflexiones que intervienen en la interrelación informativa sobre actividades, hechos, transacciones socioeconómicas que procuran medir el cumplimento de metas en diversos niveles dentro de las organizaciones sociales y entre las mismas (p. 13).

Así, la misión del contable de una empresa va más allá de la simple visión profesional instrumentalista e ingenua de la realidad, teniendo en cuenta las vinculaciones existentes entre la estructura organizativa de la empresa y los aspectos económico-financieros, ambientales y sociales. Por lo tanto, no se puede cambiar la manera de interaccionar con el medio ambiente si no se modifica la lógica de medir y revelar la relación hombre-naturaleza en la contabilidad.

Por ello se considera que existe una relación estrecha entre la situación ambiental y la contabilidad, que justifica la promoción de una estrategia pedagógica dirigida a romper el orden dominante, lo que propicia no solo la generación de conocimiento contable especializado, sino también la conjugación de otros conocimientos y saberes científicos que tienen que ver con el ambiente, en especial aquellos que se asocian a las ciencias sociales y a las ciencias naturales.

\section{Modelo para una educación ambiental renovada en la formación contable}

Una metodología de educación ambiental renovada usada en la contabilidad ambiental implica una postura opuesta a la dominante visión unidireccional. En este sentido, el contexto de aplicación del contenido de enseñanza contable debe ir más allá de la mera óptica operativa y mecanicista, conduciendo al alumnado de contabilidad a indagar acerca de los escenarios de aplicación del conocimiento contable en los campos económico, social y ambiental en las dimensiones de tiempo, espacio y contexto determinados. Así, es necesaria la reversión de la tendencia de estar ajeno a los intereses más amplios de desarrollo y de calidad de vida de la sociedad y, principalmente, de no considerar la interacción del "mundo contable" con el "mundo real", en términos de transformación y emancipación.

En la contabilidad ambiental como asignatura, es importante contextualizar su papel en la educación ecológica, resaltando la vinculación de los problemas ambientales globales a un modelo económico basado en el poder de mercado y el desarrollo plasmado en una exagerada confianza en que la ciencia y la tecnología sabrán cómo responder al agotamiento de los recursos y a los procesos de contaminación (Mora Penagos, 2009). 
Además, hay que superar la falta de una adecuada apropiación de capacitación, capaz de promover la transformación y la aplicación instrumental del conocimiento disciplinario. En otras palabras, en las formas disciplinarias hoy imperantes en la contabilidad se observa la incapacidad para definir las reglas de interdependencia y relación que están por debajo de todos los contextos de conocimiento explicitados en el proceso de formación del futuro profesional contable. Como explica Gómez Villegas (2006), la práctica no es simplemente hacer cosas, sino la transformación intencional del mundo a partir de un modelo de comprensión de este. De acuerdo con León (2008), los estudiantes de Contabilidad muestran una clara propensión a la absorción de conocimiento funcional e imaginario derivado de su campo de actuación, además de la sesgada practicidad que, en general, caracteriza la profesión contable.

Asimismo, no es suficiente explanar la preservación de la naturaleza, sino que hay que conducir a la asimilación de los principios de la ecología a través de una visión de las interrelaciones de los sistemas ecológicos, consustanciado por el contacto del alumnado con su entorno natural y social, para estimular sus capacidades perceptivas y valorativas, y proporcionarle así un aprendizaje orientado hacia un pensamiento crítico y creativo basado en nuevas capacidades cognitivas.

En la tabla 1 se plantea un modelo aproximativo del plan de asignatura para la formación contable en el campo de la educación ambiental, según el contexto de la inclusión de la dimensión ambiental de forma crítica y contextual.

Asimismo, hay que suplantar la ideología del fundamento de mercado (un desafío que ya viene siendo encarado por muchos economistas y académicos de diversas áreas). Esa crítica necesita ser contemplada de un modo más objetivo en la reflexión académica. Es consensual la creencia de que esa forma de pensar estimula la competencia, la acumulación, la expansión y la dominación, en lugar de la cooperación, la conservación y la asociación, aspectos fundamentales para la sostenibilidad de una comunidad.

Es decir, frente a las nuevas reconfiguraciones contextuales del mundo contemporáneo, existe "la necesidad de una ruptura epistemológica con las formas convencionales de enseñanza contable" (Ordóñez Norena, 2008, p. 117), que provoca "un adiestramiento en el oficio profesional de contador, sin preguntarse jamás por las relaciones de causalidad y efectividad, o por las 'lógicas' reales que dominan el mundo de la economía y los negocios" (Cruz, 2001, p. 13). Para Ordóñez (2008), esta forma convencional de proceder en la formación del contador no le permite al estudiante considerar las consecuencias de su proceder, tampoco preverlas, una vez que su instrucción se base en el hacer y no en el pensar lo que se hace.

\section{Consideraciones finales}

En este artículo se intenta mostrar que "la sostenibilidad implica que la sociedad, como sistema que hace parte del sistema natural, asuma los principios de comportamiento del sistema en el que está contenida" (Bermejo Gómez de Segura, 2007, 2011). Para eso se argumenta que el modelo tradicional de enseñanza contable sobre las cuestiones económicas, sociales y ambientales, armonizadas con el desarrollo sostenible y la naturaleza, presenta limitaciones en cuanto a no posibilitar una inclusión pedagógica universitaria valorizando el pensamiento crítico, reflexivo y propositivo vinculado a los problemas inherentes al medio ambiente. Así, la propuesta central de este artículo fue investigar la correspondencia entre la enseñanza universitaria y la educación ambiental, y se buscó formular una visión alternativa para abordar el conocimiento sobre el medio ambiente en la formación universitaria en contabilidad.

Es importante la construcción de un orden racional ambiental (que se refiere al actuar humano de acuerdo con el orden natural), donde el ser humano debe acoplarse a ese orden racional que rige la sociedad en conjunto, siendo el papel de la educación fundamental para la enseñanza frente a la racionalidad ambiental que se busca (Sabogal Aguilar y Hurtado, 2008). En otras palabras, hoy día el desarrollo económico y el medio ambiente precisan dejar de ser considerados como dos realidades antagónicas para ser tratados como complementarios (Layrargues, 1997). Teniendo en cuenta que la gran mayoría de los recursos 
naturales son finitos en su vida útil, una cuestión fundamental para la organización humana es la gestión de la escasez, mediante el uso sostenible de lo que se va consumir. Dar al mercado la prevalencia y primacía en tal decisión es un gran riesgo, a pesar de su apreciada eficacia e integridad. Nada garantiza, por ejemplo, que las empresas no tendrán conductas oportunistas al tener la capacidad de disponer del poder de mercado e imponer sus costes y condiciones de consumo. Tampoco nada ni nadie aseguran plenamente que el mercado sea un factor de eficacia, ya que no hay ninguna motivación razonable para su funcionamiento como un mecanismo de generación de igualdad. Por el contrario, puede acabar en la práctica, y esto distorsionaría más los lazos sociales y crearía externalidades (pobreza, inseguridad, intolerancia, discriminación y desigualdad) y divergencias entre el interés particular y el colectivo.

A lo largo de este ensayo teórico se plantearon algunas consideraciones generales sobre qué hacer para lograr una formación del alumnado contable desde una perspectiva humanitaria y social que incluya la vida biológica y colectiva de todas las especies, como punto central de las preocupaciones con la sociedad y la sostenibilidad. La problemática diagnosticada en esta investigación buscó sustentar los argumentos discutidos, analizando de manera descriptica las categorías de componentes importantes para la enseñanza-aprendizaje sobre el medio ambiente en la formación universitaria en contabilidad. Además, se esbozó una propuesta didáctica con cambios en la metodología de enseñanza ambiental en el campo contable.

Los resultados iniciales presentaron una oportunidad positiva para una formación contable crítica y ecológica, en la cual la educación ambiental necesitaría asumir un papel emancipador y transformador capaz de estimular una formación crítica del alumnado ante las prácticas reproductivas de las ideologías implícitas en el modelo capitalista vigente (Tozoni-Reis, 2004, 2007), ya que ellas impiden una real percepción de las causas de los problemas socioambientales y favorece la explotación de los pueblos y la expropiación de los recursos locales por el capital internacional.

Las evidencias ofrecidas en este estudio posibilitaron un puente entre esas dos perspectivas, educación ambiental y enseñanza contable, y demostraron que es responsabilidad de cada uno dentro de la universidad profundizar en el análisis de la realidad y seguir implementando la evolución de la conciencia a nivel personal.

Igualmente, es preciso superar a los posibles miedos arraigados y actuar en cada situación para contribuir a la corrección del rumbo de la academia. Con esa postura ya se estará obrando en la construcción de una nueva realidad social y económica en la dimensión ambiental, sin exaración de los recursos naturales del planeta.

La investigación fue relevante porque visibilizó la necesidad de profundizar en la comprensión de los mecanismos políticos que subyugan los intereses de calidad de vida y bienestar de las poblaciones locales en su desarrollo socioeconómico y su preservación ambiental a los intereses escudos de concentración de poder y la riqueza. De acuerdo con Fay (1987), hay que promover análisis y proponer alternativas y medidas para que los seres humanos sean iluminados, empoderados y emancipados. Algo similar se puede hacer a través de cambios en las prácticas y políticas consideradas irracionales u opresivas, con un examen crítico de los aspectos sociales, económicos y políticos contemporáneos, a través de una evaluación racional de la situación de vida de las personas y de los acuerdos sociales.

Por último, se recomiendan nuevas investigaciones dirigidas a la consolidación de una masa crítica de pensamiento, comprometida con una reflexión teórica contable de contenido humano y social, vinculando la sustentabilidad y el desarrollo sostenible como centro de la preocupación académica, conforme a las ideas discutidas en la presente investigación. 
Tabla 1. Aspectos básicos relacionados con la educación ambiental en contabilidad

\section{Campos de intervención Temas centrales de estudio}

\section{Ejes articuladores}

Contexto de justificación

Enfoque relativo a cuestiones de validación para confirmar la autenticidad de una producción, la veracidad de una creencia 0 la aceptabilidad de una teoría para construir evidencias que puedan dar soporte para las afirmaciones y el incremento del conocimiento contable disponible en este campo.
Identidad disciplinaria

Se refiere al objeto de estudio de la contabilidad socioambiental, la metodología empleada, el discurso de legitimación y las evidencias que el conocimiento epistemológico y los intereses dominantes imponen en el ámbito social y ambiental.
Lenguajes de comunicación contable ambiental

- Objeto y método en la contabilidad ambiental.

- Enfoques de pensamiento en contabilidad ambiental.

- Intereses dominadores del conocimiento contable ambiental.

- Paradigmas y programas de investigación ambiental.

Teorías de representación contable ambiental

- Representatividad de la contabilidad ambiental y sus estructuras.

- Problemas inherentes a las estructuras de medición del medio ambiente.

- Problemas que comportan la valoración del medioambiente.

- Problemas pertinentes a la revelación de los activos y pasivos ambientales.

- Obstáculos a un modelo contable socioambiental globalizado.

Contexto de descubrimiento
Comprende la invención y
la producción de hipótesis,
teorías, hallazgos y conceptos
relacionados con las
circunstancias históricas,
psicológicas, sociológicas,
políticas, económicas,
axiológicas y tecnológicas que
contribuyen a su formulación
en un tiempo y lugar
determinados.

\section{Competencia disciplinar}

Se entiende como

la capacidad de articulación con los diferentes componentes y elementos que componen la estructura del conocimiento en el contexto social y ambiental.
Contabilidad de gestión socioambiental

- Sistema de información contable ambiental.

- Revelación de la información socioambiental.

\section{Herramientas para la gestión de la sustentabilidad}

- Responsabilidades y daños ambientales.

- Desarrollo y medio ambiente sustentable.

Integración de los informes sociales y ambientales

- Responsabilidad social de la empresa.

- Mecanismos de auditoría social.

Disponibilidad
interdisciplinar
Se concibe a través
de la articulación e
integración con otras
áreas transversales de
conocimiento.
conocimiento.

\author{
Abordaje económico \\ - Teorías económicas dominantes. \\ - Impactos de los modelos económicos. \\ - Economía del medio ambiente.
}

Mecanismos de gestión y administración

- Organización de los recursos naturales.

- Gestión del capital humano.

- Contribuciones de la innovación y de la tecnología.

Ámbito jurídico y legal

- Internacionalización empresarial.

- Regulación jurídica en el campo ambiental.

Concepción ética y moral

- Código de ética ambiental.

- Discurso ético empresarial.

\section{Componente político y social}

- Desigualdades y políticas públicas.

- Corrupción en las relaciones de poder.

Fuente: elaboración propia, a partir de datos de León (2008). 


\section{Referencias}

Abela, D. J. (2000). Las técnicas de Análisis de Contenido: una revisión actualizada. Fundación Centro Estudios Andaluces. Universidad de Granada, 10(2), 1-34.

Arntz, W., Chasse, B. y Vicente, M. (2006). ¿iY tú qué sabes!? Descubre las infinitas posibilidades para cambiar tu realidad cotidiana. (Trad. E. Gómez-Acebo Príes y C. González del Yerro). Madrid, España: Palmyra Libros. Recuperado de https://fisicartes.files.wordpress. com/2017/01/y-tu-que-sabes.pdf.

Banco Mundial (2008). The Growth Report: Strategies for Sustained Growth and Inclusive Development. Recuperado de https://openknowledge.worldbank.org/handle/10986/6507

Bardin, L. (2016). Análise de conteúdo. (L. A. Reto y A. Pinheiro, Trad.) (4ta. Ed). Lisboa:, Portugal: Edições 70 .

Bermejo Gómez de Segura, R. (2007). El paradigma dominante como obstáculo para la sostenibilidad. La transformación epistemológica y paradigmática de la economía sostenible. Ekonomiaz: revista vasca de economía, 64(1), 36-71. Recuperado de https://dialnet. unirioja.es/servlet/articulo?codigo $=2350095$

Bermejo Gómez de Segura, R. (2011). Manual para una economía sostenible. Madrid, España: Los Libros de la Catarata.

Bomfim, A. M. (2011). Trabalho, Meio Ambiente e Educação: apontamentos à Educação Ambiental a partir da Filosofia da Práxis. Revista Labor, 1(5), 3-19. Recuperado de http://www.periodicos.ufc.br/labor/article/ view/6635

Bonfim, A. M. (2011). Educação ambiental para além do capital: balanço de estudos e alguns apontamentos à EA sob a perspectiva do Trabalho - GPTEEA (IFRJ). Trabalho Necessário, 9(13), 1-20. doi: https://doi. org/10.22409/tn.9i13.p6849

Capra, F. y Luisi, P.L. (2014). A visão sistêmica da vida: uma concepção unificada e suas implicações filosóficas, políticas, sociais e econômicas. São Paulo, Brasil: Cultrix.

Carson, R. (1962). Primavera silenciosa. (2da. Ed.). São Paulo: Edições Melhoramentos. Recuperado de https://biowit.files.wordpress.com/2010/11/primavera_silenciosa_-_rachel_carson_-_pt.pdf

Cartas de Cristo. (2012). A Consciência Crística manifestada. (2da. Ed). São Paulo, Brasil: Almenara Editorial.

Chanlat, J.-F. (2002). Ciencias Sociales y Administración: en defensa de una antropología general. Medellín, Colombia: Fondo Editorial Universidad Eafit.

Collado-Ruano, J. (2017). Educación y desarrollo sostenible: la creatividad de la naturaleza para innovar en la formación humana. Educación y Educadores, 20(2), 229-248. doi: http://dx.doi.org/10.5294/edu.2017.20.2.4

Cosenza, J. P., Ribeiro, C. M. y Dios, S. A. (2017). Companies' globalization and social problems: the role of Corporate Social Responsibility. En Corporate Responsibility Research Conference. University of Seville, Sevilla, España.

Cosenza, J. P., Cardoso Teixeira, A. y De Souza Sant'Anna, R. (2012). Reflexão Sobre Relações Entre Poder e Contabilidade. Contabilidade, Gestão e Governança, 15(2), 78-94. Recuperado de https://cgg-amg.unb.br/index. php/contabil/article/viewFile/429/pdf

Cruz, F. (2001). Curiosidad, investigación, espíritu crítico. En Memorias del XV Congreso Nacional de Estudiantes de Contaduría Pública (pp. 9-15). Cali, Colombia: Federación Nacional de Estudiantes de Contaduria Publica de Colombia (Fenecop), Asociación de Estudiantes de Contaduría Pública de la Universidad del Valle (Asecuva), Universidad del Valle.

Deluiz, N. y Novicki, V. (2004). Trabalho, meio ambiente e desenvolvimento sustentável: implicações para uma proposta de formação crítica. Boletim Técnico do Senac, 30(2), 19-29. Recuperado de http://www.bts.senac.br/index.php/bts/article/view/516/439

Dias, B.C. (2013). Em busca de uma práxis em educação ambiental crítica: contribuições de alguns pesquisadores do Brasil. (Tesis de maestría). Instituto Federal de Educação, Ciência e Tecnologia do Rio de Janeiro, Nilópolis, Brasil.

Dias, B. C. y Bomfim, A. M. (2011). A “teoria do fazer" em educação ambiental crítica: uma reflexão construída em contraposição à educação ambiental conservadora. Recuperado de http://www.nutes.ufrj.br/abrapec/viiienpec/resumos/R0098-1.pdf

Emoto, M. (1999). The Message from Water. Tokyo, Japón: Hado Kyoiku Sha.

Fay, B. (1987). Critical social science. Cambridge: Polity Press.

Garay Salamanca, L. J., Salcedo-Albarán, E., León-Beltrán, I. y Guerrero, B. (2008). La captura y reconfiguración cooptada del Estado en Colombia. Bogotá: Fundación Método, Fundación Avina, Transparencia por Colombia.

García Casella, C.L. y Rodríguez de Ramírez, M. del C. (2001). Elementos para una teoría general de contabilidad. Buenos Aires, Argentina: La Ley.

Gómez Patiño, D. P. (2011). El pacto global de las Naciones Unidas: sobre la responsabilidad social, la anticorrupción y la seguridad. Prolegómenos, 14(28), 217-231, doi: https://doi.org/10.18359/prole.2388 
Gómez Villegas, M. (2006). Comentarios sobre el aprendizaje-construcción de la teoría contable. Lúmina, (7), 129-153. Recuperado de revistasum.umanizales.edu. co/ojs/index.php/Lumina/article/download/1180/1256/

Guimarães, M. (2000). Educação ambiental: no consenso um debate? Campinas, Brasil: Papirus.

Layrargues, P. P. (1997). Do ecodesenvolvimento ao desenvolvimento sustentável: evolução de um conceito? Proposta, 24(71), 1-5. Recuperado de https://fase.org. br/wp-content/uploads/2016/06/Proposta-Revista-Trimestral-de-Debate-da-Fase-n\%C2\%BA-71-1997-02.pdf

Leff, E. (1994). Ecología y capital. Racionalidad ambiental, democracia participativa y desarrollo sustentable. Ciudad de México, México: Siglo xxi.

Leff, E. (1995). ¿De quién es la naturaleza? Sobre la reapropiación social de los recursos naturales. Gaceta Ecológica, (37), 28-35.

Leff, E. (2002). Epistemologia Ambiental. (S. Valenzuela, Trad.) (5ta. Ed.). São Paulo, Brasil: Cortez.

Leff, E. (1996). Los nuevos actores sociales del ambientalismo en el medio rural. En H. C. Grammnt y H. T. Gaona (Coords.), La sociedad rural mexicana frente al nuevo milenio. Vol. IV (pp. 1-3). Ciudad de México, México: Plaza y Valdéz.

Leff, E. (2008). Educação ambiental e desenvolvimento sustentável. En M. Reigota (Org.) Verde cotidiano: o meio ambiente em discussão (3ra. Ed.) (pp. 97-112). Petrópolis, Brasil: DP et Alii.

Leis, H. R. (1991). Ecologia e política mundial. Río de Janeiro, Brasil: Editora Vozes.

León Martínez, G. (2008). La resignación de los 'contextos del conocimiento': a propósito del Plan de Formación Contable. En Perspectivas críticas de la contabilidad: reflexiones y críticas contables alternas al pensamiento único. Memorias del VII Simposio Nacional de Investigación Contable y Docencia (pp. 127-144). Bogotá, Colombia: Universidad Nacional de Colombia.

Loureiro, C. F. B. (2004). Trajetória e Fundamentos da Educação Ambiental. São Paulo, Brasil: Cortez.

Loureiro, C. F. B. (2007). Pensamento Crítico, tradição marxista e a questão ambiental: Ampliando os debates. En C. F. B. Loureiro (Org.), A questão ambiental no pensamento crítico: natureza, trabalho e educação (pp. 13-67). Río de Janeiro, Brasil: Quartet.

Mora Penagos, W. M. (2007). Respuesta de la universidad a los problemas socioambientales: la ambientalización del currículo en la educación superior. Investigación en la Escuela. (63), 65-76. Recuperado de https://revistascientificas.us.es/index.php/IE/article/view/7181/6354

Mora Penagos, W. M. (2009). Educación ambiental y educación para el desarrollo sostenible ante la crisis planetaria: demandas a los procesos formativos del profesorado. Tecné, Episteme y Didaxis, (26), 7-35, doi: https://doi.org/10.17227/ted.num26-416

Mora Penagos, W. M. (2012). Ambientalización curricular en la educación superior: un estudio cualitativo de las ideas del profesorado. Profesorado. Revista de Currículum y Formación de Profesorado, 16(2), 77-103. Recuperado de https://www.redalyc.org/ pdf/567/56724395006.pdf

Moreno Martínez, E. (2016). Presentación. En R. Peréz Garcés, L. Victorino Ramírez y M. L. Quintero Soto (Coords.), Educación Ambiental y Sociedad: sabers locales para el desarrollo y la sustentabilidad (pp. 1-3). Ciudad de Mexico, México: Laberinto Ediciones.

Organización para la Cooperación y el Desarrollo Económicos (OECD). (2011). Tools for Delivering on Green Growth. París, Francia: autor. Recuperado de https:// www.oecd.org/greengrowth/48012326.pdf

Organización de las Naciones Unidas para la Educación, la Ciencia y la Cultura (Unesco) (1997). Conferencia Intergubernamental sobre Educación Ambiental, Tbilisi, URSS, 14-26 de octubre de 1977: informe final. United Nations Environment Programme. París, Francia. Recuperado de https://unesdoc.unesco.org/ark:/48223/ pf0000032763_spa

Organización de las Naciones Unidas para la Educación, la Ciencia y la Cultura (Unesco). (1980). La educación ambiental. Las grandes orientaciones de la Conferencia de Tbilisi. París, Francia: Organización de las Naciones Unidas para la Educación, la Ciencia y la Cultura.

Organización de las Naciones Unidas para la Educación, la Ciencia y la Cultura (Unesco) y Programa de las Naciones Unidas para el Ambiente (UNEP). (1985). Interdisciplinary Approaches in Environmental Education. París, Francia: Division of Science, Technical and Environmental Education.

Organización de las Naciones Unidas para la Educación, la Ciencia y la Cultura (Unesco). (2014). El Desarrollo sostenible comienza por la educación: cómo puede contribuir la educación a los objetivos propuestos para después de 2015. París, Francia: autor. Recuperado de https:// unesdoc.unesco.org/ark:/48223/pf0000230508_spa

Organización de las Naciones Unidas para la Educación, la Ciencia y la Cultura (Unesco). (2017). Educación para los Objetivos de Desarrollo Sostenible Objetivos de aprendizaje. París, Francia: Organización de las Naciones Unidas para la Educación, la Ciencia y la Cultura. Recuperado de https://unesdoc.unesco.org/ ark:/48223/pf0000247444

Ordóñez Norena, S. L. (2008). Contra el adiestramiento contable: invitación a la ruptura epistemológica en la 
formación del contador público. En Perspectivas críticas de la Contabilidad: reflexiones y crítica contables alternas al pensamiento único. Memorias del VII Simposio Nacional de Investigación Contable y Docencia (pp. 117-126). Bogotá, Colombia: Universidad Nacional de Colombia.

Pérez, G. (1994). Investigación cualitativa. Retos e interrogantes. (Tomo II). Madrid, España: La Muralla S. A.

Pérez Garcés, R., Victorino Ramírez, L. y Quintero Soto, M. L. (Coords.). (2016). Educación ambiental y sociedad: saber locales para el desarrollo y la sustentabilidad. Ciudad de México, México: Laberinto Ediciones.

Quinche Martín, F. L. (2017). Una mirada crítica a las teorías predominantes de la responsabilidad social corporativa. Revista de la Facultad de Ciencias Económicas, 25(2), 159-178, doi: https://doi.org/10.18359/rfce.3071

Rôças, G. y Anjos, M. B. (2014). Reflexões sobre a prática e a teoria da Educação Ambiental. Rio de Janeiro, Brasil: Publit Soluções Editoriais.

Rodríguez Villamil, H. y Yolanda M. Guerra, Y. M. (2009). Propuesta de educación para el desarrollo sostenible: perspectiva pedagógica para la Universidad Militar Nueva Granada. Revista Educación y Desarrollo Social, 3(2), 72-85. Recuperado de https://revistas.unimilitar. edu.co/index.php/reds/article/view/879/630

Rodríguez Villamil, H., Guerra García, Y. M. y Guzmán Cómbita, A. (2011). El rol de la educación frente al desarrollo sostenible: una mirada desde el marco del decenio de la educación para el desarrollo sostenible 2005-2014. Revista Educación y Desarrollo Social, 5(1), 127-138. Recuperado de www.umng.edu.co/documents/63968/80124/9.pdf

Rojas López, M.D., Uribe Giraldo, L.M. y Rojas Valencia, M. F. (2018). Educación para el desarrollo sostenible caso Universidad Nacional de Colombia - sede Medellín (pp. 249-258). En M. L. Quintero Garzón M. D. Sánchez Fernández (Comps.), Responsabilidad social corporativa: una mirada integral en América Latina. Cali, Colombia: Universidad del Valle. Recuperado de http://hdl.handle.net/10893/10841

Sabogal Aguilar, J. y Hurtado, H. (2008). Elementos del concepto racionalidad ambiental. Revista de la Facultad de Ciencias Económicas, 16(2), 117-132. Recuperado de http://www.redalyc.org/articulo.oa?id=90916208

Santos, B. S. (2010). Um discurso sobre as ciências. Oporto, Portugal: Edições Aforamento.

Silva, P. R. (2019). Economia, consciência e abundância (2da. Ed.). Niterói, Brasil: Bambual Editora.

Stiglitz, J. (2006). Como hacer que funcione la globalización. Madrid, España: Taurus.

Tirole, J. (2018). Economia do Bem Comum. Lisboa, Portugal: Guerra e Paz Editores.

Tozoni-Reis, M. F. de C. (2004). Educação ambiental: natureza, razão e história. Campinas, Brasil: Autores Associados.

Tozoni-Reis, M.F. de C. (2007). Fundamentos Teóricos para uma pedagogia da educação ambiental: algumas contribuições. En 30a Reunião Anual da ANPEd, Caxambu, Brasil.

United Nations Environment Programe (PNUMA) (2011). Decoupling Natural Resource Use and Environmental Impacts from Economics Growth. United Nations Environment Programe (UNEP). Recuperado de http:// wedocs.unep.org/handle/20.500.11822/9816

Vásquez Vargas, M. J. (2014). Educación para el desarrollo sostenible (EDS) - un posicionamiento de jóvenes progresistas en América Central. Perspectivas, (7). Recuperado de https://library.fes.de/pdf-files/bueros/ fesamcentral/11121.pdf

Veiga, J. E. da. (2012). Economia em transição. En F. Almeida (Org.), Desenvolvimento Sustentável - 2012 a 2050 (recurso eletrônico): visão, rumos e contradições (pp. 3-14). Río de Janeiro, Brasil: Elsevier. 